
\title{
28 Research Soure \\ Radiative Cooling Sorbent towards High \\ Performance All Weather Ambient Water Harvesting
}

Wenkai Zhu

Purdue University

Chi Zhang

ETH

Yun Zhang

Purdue University

Xiwei Shan

Purdue University

Akshay Rao

Purdue University

Sarah Pitts

Purdue

Travest Woodbury

Purdue

Dominique Derome

Université de Sherbrooke

David Warsinger

Purdue University

Lisa Mauer

Purdue

\section{Xiulin Ruan}

Purdue University https://orcid.org/0000-0001-7611-7449

Jan Carmeliet

ETH

Tian Li ( $\nabla$ tianli@purdue.edu )

Purdue University https://orcid.org/0000-0002-1087-0662

\section{Article}

Keywords: cellulose, salt, radiative cooling, sorbent, atmospheric water harvesting, sustainability, large scale

Posted Date: February 28th, 2022 
DOI: https://doi.org/10.21203/rs.3.rs-1394191/v1

License: (c) (1) This work is licensed under a Creative Commons Attribution 4.0 International License. Read Full License 


\section{Abstract}

Emerging atmospheric water harvesting $(\mathrm{AWH})$ technologies promise water supply to underdeveloped regions that have no access to liquid water resources. The prevailing AWH systems, including condensation- or sorption-based, mostly rely on a single mechanism and thus have a limited range of working conditions and inferior performance. In this study, we synergistically integrate multiple mechanisms, including thermosorption effect, radiative cooling, and multiscale cellulose-water interactions, and demonstrate a low-cost $\left(\sim 4\right.$ USD kg-1) and high performance (up to $6.75 \mathrm{~L} \mathrm{~kg}^{-1} \mathrm{day}^{-1}$ in field tests) AWH system requiring zero active energy input. The proposed system consists of a highly scalable and sustainable cellulose scaffold impregnated with hygroscopic deliquescent lithium chloride $(\mathrm{LiCl})$ salt. Cellulose scaffold and coated $\mathrm{LiCl}$ synergistically interact with water at different scales from molecular, to nanometer, and micrometer scales, providing a fast harvesting rate and high yield over a wide operation range. Iterating radiative cooling and solar heating workflow achieves simultaneous enhancement of water capture and release through the so-called temperature-swing strategy. The captured water in return facilitates radiative cooling due to the intrinsically high infrared (IR) emissivity of the LiCl-cellulose composite. With our simple yet effective material design, the AWH working range extends to lower than $\sim 10 \%$ relative humidity $(\mathrm{RH})$, and the water uptake in the controlled lab test reaches $16 \mathrm{~kg} \mathrm{~kg}^{-1}$ at $90 \% \mathrm{RH}$ (sample at $19^{\circ} \mathrm{C}$, i.e. $6^{\circ} \mathrm{C}$ below $25^{\circ} \mathrm{C}$ ambient temperature). In addition, we propose a theoretical model capable of elucidating the experimental water uptake curves and demonstrating the synergy among cellulose-LiCl-water-energy interaction. The promising performance emphasizes the potential of involving multiple AWH mechanisms and points to an alternative pathway to stimulate future improvement.

\section{Introduction}

About half of the world population, i.e. 4 billion people, undergo severe water scarcity at least seasonally, posing a systematic threat to humanity (1). Technologies, such as thermal desalination (2-7) and solar steam generation (8-10), are being developed and used to mitigate the global freshwater crisis. However, these conventional approaches demand intense energy input and, most importantly, access to water resources, such as precipitation, surface- or groundwater, or coastal water, limiting their applicability. In search of geographically independent water collection, atmospheric water harvesting $(\mathrm{AWH})$ emerges as a promising candidate to harvest freshwater with drinking water quality and potential scalability.

Atmospheric water vapor and droplets account for $~ 10 \%$ of the global freshwater reservoir $(11,12)$. There are tremendous efforts in developing novel AWH systems, most of which can be categorized as either condensation- or sorption-based devices. Condensation-based AWH systems rely on cooling or mechanical compressing to induce vapor-to-liquid phase transition. The major drawbacks of condensation include the requirement for high relative humidity $(\mathrm{RH})(13,14)$, e.g. fog harvesting, and/or sizeable energy input for cooling $(15,16)$, which are not viable for arid and developing regions. In contrast to condensation, sorption-based AWH systems can work over a much wider $\mathrm{RH}$ range exploiting the 
hygroscopicity of adsorbents. Typical sorption materials include desiccator salts (17-19), metal-organic frameworks $(20-22)$, and thermo-responsive gels $(23,24)$. Strong water affinity contributes to water capture but hampers subsequent water release. Yet to be resolved is the long-standing quest for simultaneous enhancement of water capture and release, two processes difficult to optimize simultaneously. Sorption materials that are fully sustainable, cost-effective, and scalable are lacking. Previous endeavors have focused on individual mechanisms but have yet to fully exploit the potential of natural materials with hierarchical structures. Only limited success has been achieved on AWH devices within a wide working range and addressing the intrinsic conflict between water capture and release (2528).

This study aims to tackle the above-mentioned challenges by synergistically integrating multiple mechanisms, including thermosorption effect, radiative cooling, and multiscale cellulose water interaction, forming a highly integrated protocol that, to the best of our knowledge, has not yet been explored in full detail. For the first time, we demonstrate the fundamental interplay of key components in a dual-mechanism AWH system by a LiCl-cellulose radiative cooling composite and document its promising performance at both low and high $\mathrm{RH}$. Moreover, we investigate the temperature-swing strategy, i.e. triggering water capture and release by switching the system temperature to be hot and cold, respectively, to simultaneously achieve high water capture and release. Lab experiments, field tests, and theoretical modeling are conducted to elaborate how the microscopic LiCl-cellulose-water coupling and the environmental factors synergistically affect the macroscopic material performance. The theoretical model is cross-validated with the experimental water uptake experiments with or without radiative cooling and predicts water uptake within a range beyond the current experimental limits. An outdoor batch-mode AWH device demonstrates water capture (water uptake) as high as $6.75 \mathrm{~L} \mathrm{~kg}^{-1}$ day $^{-1}\left(70 \% \mathrm{RH}, 21.6^{\circ} \mathrm{C}\right)$ and water production up to $5.97 \mathrm{~L} \mathrm{~kg}^{-1}$ day $^{-1}$ under $0.9-1.1$ sun after eight continuous capture-release cycles with a cost as low as 4.05 USD kg-1 (cellulose: $1.5 \mathrm{USD} \mathrm{kg}^{-1}$, salt: $10 \mathrm{USD} \mathrm{kg}^{-1}$ (29)). The efficient and high-performance AWH composite operating over a wide of the $\mathrm{RH}$ spectrum opens new opportunities for both fundamental research and practical applications to account for the seasonal and climate variation of the $\mathrm{RH}$, especially in arid areas (such as the Death Valley), where $\mathrm{RH}$ varies between $5 \%$ and $95 \%$ most years (30).

Cellulose, one of the most abundant biopolymers on earth, has many unique functional properties including robust mechanics, strong hydrophilicity, carbon-neutrality, etc. $(31,32)$. Moreover, in recent studies the radiative cooling effect of cellulose $(33,34)$ is demonstrated, a mechanism which gives rise to several new applications. Cellulose materials emit thermal radiation, and when facing the sky, the emitted radiation escapes the earth through the atmospheric transparent window $(8-13 \mu \mathrm{m})$ and ends up in the ultracold universe (Fig. 1A) (35). The emitted heat exceeds the adsorbed solar heat resulting in a net cooling effect at zero energy cost. Cooling is highly beneficial for AWH because it not only facilitates the exothermic sorption process, but also lowers the saturation vapor pressure which consequently promotes condensation. Radiative cooling and sorption form a positive feedback loop. Cooling promotes sorption while the adsorbed water molecules contribute to an even higher cooling power due to their high IR 
emissivity. Though cellulose is hygroscopic and can adsorb up to 40\% water content by weight, it is not an ideal AWH material alone. The cellulose scaffold can be impregnated with $\mathrm{LiCl}$, one of the most hygroscopic salts at a low $\mathrm{RH}$, to boost its water harvesting ability. $\mathrm{LiCl}$ salt adsorbs limited amounts of water at low $\mathrm{RH}$ conditions $(<5 \% \mathrm{RH})$, but when the $\mathrm{RH}$ is raised above $11 \%$, the deliquescence point $\left(\mathrm{RH}_{\mathrm{o}}\right)$ of $\mathrm{LiCl}$, the salt will deliquesce, forming a saturated solution that can ultimately take up more than $1000 \%$ water content as the $\mathrm{RH}$ is elevated further $(36,37)$. $\mathrm{LiCl}$ desiccant is also advantageous in terms of water release, as $\mathrm{LiCl}$ regenerates at $40^{\circ} \mathrm{C}(38)$, a temperature readily obtainable via solar heating. Impregnation of $\mathrm{LiCl}$ in the cellulose multiscale hierarchical pore network extends the working $\mathrm{RH}$ range and boosts the water harvesting capacity and kinetics, compared to salt-alone and cellulose-alone systems. The cellulose scaffold, in return, structurally supports $\mathrm{LiCl}$ and retains the water and $\mathrm{LiCl}$ solution (Fig. 1B). The aggregation, agglomeration, and deliquescence commonly observed in $\mathrm{LiCl}$ powder (Fig. 1C) are alleviated in the cellulose scaffold for a non-degraded sorption capability $(18,37$, 39-41). The hydrated LiCl-cellulose composite increases optical transparency across the solar spectrum and allows rapid water evaporation by solar heating with a dark substrate underneath. Cycles of radiative cooling and solar heating shift the system temperature between low and high favoring water capture and release respectively, i.e. the temperature-swing strategy. This yields an exceedingly high harvesting capacity which has been one of the major bottlenecks in conventional ambient water harvesting systems.

To fabricate the LiCl-cellulose composite, the cellulose scaffold $(0.574 \mathrm{~mm}$ thickness fabric) is immersed into $\mathrm{LiCl}$ solution and then oven-dried (Note S1). The LiCl ions gradually intercalate into the cellulose fiber matrix during the thrice dipping-drying steps, as shown in Fig. 2A. Following the oven drying process, the well-dispersed ions recrystallize and bind tightly to the cellulose surfaces. The cellulose scaffold can hold up to $50 \mathrm{wt} \%$ of the $\mathrm{LiCl}$ salt with perfectly retaining the solution (Fig. S4). The high aspect ratio of the fiber bundle is composed of multiscale features including microscale fiber bundles $\sim 20 \mu \mathrm{m}$ in diameter to nanofibers $\sim 20 \mathrm{~nm}$ in diameter (Fig. 2B-D, Fig. S2). The LiCl brine solution quickly infiltrates the cellulose scaffold and the salt ions achieve nanoscale dispersion, as indicated by the energy-dispersive Xray spectroscopy (EDX) mappings of fiber networks and cross-sections (Fig. 2E-H, Fig. S5). The hydrophilic surface as well as the numerous channels between fibers and fiber bundles, revealed in the scanning electron microscope (SEM) images (Fig. 2B-H), facilitate the salt solution infiltration process. The intrinsically hydrophilic and highly porous fiber network efficiently captures and stores water. Our solution-based salt impregnation process can be easily incorporated into the well-established cellulosebased paper, textile, and membrane manufacturing process. All these enable the fast deployment of the proposed device, opening industrialization opportunities.

Radiative cooling is a systematic outcome of photonic and vibrational interactions between the multiscale structure of cellulose, solar radiation, and the ambient environment (Fig. 3A). Figure 3B exhibits the emittance over the wavelength range $250 \mathrm{~nm}-20 \mu \mathrm{m}$, i.e. solar spectrum and sky transmittance spectrum (IR range), of the cellulose scaffold and LiCl-cellulose composite in dry and wet states (Note S3). In the visible and near-IR spectra, the emittance is found to be low as more than $95 \%$ of incident radiation with wavelength between $0.4-1.5 \mu \mathrm{m}$ is intensely backscattered by the nanofibers and 
microfibers at the corresponding length scale. The microfibers and larger fiber bundles $(2-20 \mu \mathrm{m})$ contribute to amplifying and broadening the emittance peaks to increase the radiative energy leaving the composite. The particularly strong IR radiation of cellulose stems from the multimode vibrations of molecular chains, including $\mathrm{C}-\mathrm{O}$ stretching and $\mathrm{O}-\mathrm{H}$ stretching (42), where the emission wavelengths align with the atmospheric sky window (8-13 $\mu \mathrm{m})$, permitting energy dissipation to deep space. The aforementioned light-matter interactions provide the LiCl-cellulose composite a selective emittance spectrum that allows a spontaneous and rapid cooling to sub-ambient surface temperature during nighttime (43). Wet samples emit more strongly in the IR range than dry ones because the additional hydrogen bonding between the $\mathrm{OH}^{-}$branches and $\mathrm{H}_{2} \mathrm{O}$ molecules complements the missing molecular vibration modes and water occupies the original pore space providing more emitting substances. Also, bulk water itself is a good IR emitter with near-unity emissivity (44). These clearly suggest that sorption and radiative cooling mutually enhance each other, forming a positive feedback loop, a merit of system design. The effective emissivity over the atmospheric window is 0.75 for the cellulose scaffold in the dry condition and is enhanced to 0.80 when coated with $\mathrm{LiCl}$.

Field tests are conducted to demonstrate the functionality of the AWH composite in real environments. The field test apparatuses are illustrated in Fig. S9-10. Water harvesting tests are carried out during nights from Nov. 2020 to May 2021 in West Lafayette, IN $\left(40.4237^{\circ} \mathrm{N}, 86.9212^{\circ} \mathrm{W}\right)$. Radiative cooling effectively reduces sample surface temperature to $6.0-8.7^{\circ} \mathrm{C}$ below ambient temperature, as shown by the representative data of Apr. 1st, 2021 and Nov. 11th, 2020 at an RH range of 48\%-82\% (Fig. 3C). The sporadic temperature surge in Fig. $3 \mathrm{C}$ is caused by manual measurement of sample weight. The radiative cooling power measured by a feedback heater (Fig. 3D) exhibits an average cooling power of $110 \mathrm{~W} \mathrm{~m}^{-2}$ for LiCl-cellulose composite during the night of May 14th, 2021 and $82.3 \mathrm{~W} \mathrm{~m}^{-2}$ for the cellulose scaffold during the night of Jan. 21st, 2021. The cooling power difference is a consequence of different climate conditions. The LiCl-cellulose composite shows a higher cooling power when the test is conducted under a higher ambient temperature and low absolute humidity during the summer. We present a theoretical model to estimate the cooling power at different ambient temperatures based on the measured optical emittance of the three samples, wherein the wet composite shows the highest cooling power while dry cellulose scaffold the lowest (Note S4). Rapid water release is achieved by placing a dark substrate underneath the LiCl-cellulose composite during the daytime (Fig. 3E). The LiCl-cellulose composite transmits more sunlight when it is wet (Fig. S6) because of the better match of the refractive indices of cellulose and water than cellulose and air. The wet composite on a dark substrate has a higher solar absorption of $67.7 \%$ to facilitate water release (Fig. 3F). The numerous channels and fiber surfaces serve as paths for water transport from inside to the composite surface enhancing the drying process and release of stored water. The outdoor cellulose scaffold demonstrates a lower surface temperature of the composite due to radiative cooling, with exposure to direct solar irradiation, as shown in Fig. 3G.

To further elucidate the working mechanism of the LiCl-cellulose composite, we individually analyze and decouple the synergistic effect of the radiative cooling, hygroscopic $\mathrm{LiCl}$, and cellulose fibril structure (Fig. 4A). At atomistic scale, the water dipole attracts the counter-ions and breaks the ionic bonds of $\mathrm{LiCl}$ 
leading to its dissolution. Meanwhile, the polar hydroxyl groups on cellulose chains hydrogen bond strongly with polar water molecules. The multiscale pore structure of the cellulose scaffold facilitates water harvesting with micropore filling $(<2 \mathrm{~nm})$, mono/multilayer adsorption on pore surfaces and capillary condensation in mesopores $(>4 \mathrm{~nm}$ ). In nanoscale pores the intermolecular interactions of the surfaces overlap thus creating strong attraction promoting nucleation. The adsorption can occur even at low RH. The fibers at this scale are cellulose aggregates. On the mesopore scale, a large volume of water is retained via capillary forces. Capillary condensation happens at sub-saturation vapor pressure, as described by the Kelvin equation. The porous structure at this scale denotes larger scale fiber assemblies. Larger scale structures, such as the fiber network, transport water by wicking in the inter-fiber pores spaces, as also occurs in desert plants (45). Single fibers consist of a bundle of cells. The enhanced water capturing performance stems from four mechanisms (Fig. 4B): 1. Physisorption of cellulose; 2. Chemisorption of $\mathrm{LiCl}$, i.e. forming $\mathrm{LiCl}$ hydrates; 3 . Sorption of water vapor into $\mathrm{LiCl}$ solution (here called 1st condensation), occurs when $\mathrm{RH}$ is higher than the $\mathrm{RH}_{0} ; 4$. Condensation of water vapor due to vapor saturation (here called 2nd condensation). Each of these mechanisms can be described by corresponding physical models. The physisorption is frequently represented by the Guggenheim-Anderson-de Boer (GAB) sorption model. The hydration of $\mathrm{LiCl}$ can be described by its phase diagram $(36,46)$. Isolated water condensation (2nd condensation) is a (quasi) linear function of time assuming heat and mass transfer reach equilibrium. With these considerations, a theoretical model for the sorption of the composite is developed as the weighted summation of the four mechanisms (Note S7).

Controlled experiments are conducted in an environmental chamber. The working mechanisms are identical to the field test but with precise control of the sample temperature, ambient temperature, and $\mathrm{RH}$ for a systematic performance evaluation (Note S8). Figure 4C displays the water uptake curves of at a $6^{\circ} \mathrm{C}$ temperature drop from $25^{\circ} \mathrm{C}$ ambient temperature, $10-30 \mathrm{wt} \% \mathrm{LiCl}$ content. At $\sim 8 \% \mathrm{RH}$, sorption surges due to the onset of salt deliquescence (Fig. 4C). Sorption dominates the water uptake until RH > $60 \%$ where condensation commences and dominates. The theoretical model quantitatively agrees with the experiments, as shown in Fig. 4C. Outcome of this model also clearly demonstrates that radiative cooling substantially improved the water capture (Fig. S11-13). The model allows to derive a phase diagram relating $\mathrm{LiCl}$ content, required $\mathrm{RH}$ and resulting water uptake as shown in Fig. 4D. The phase diagram breaks down in two regimes where one of the two mechanisms dominates performance. In subdew point temperature conditions, uptake is driven by the available power to cool the scaffold compensating for the latent heat transfer due to condensation. Whereas above the dew point, the water uptake is adsorption driven. More details are given in Note S9. The lab tests reveal a 2-fold and 9-fold increase of water uptake solely due to the $6^{\circ} \mathrm{C}$ cooling of the composite at $30 \%$ and $70 \% \mathrm{RH}$ respectively (Fig. 4E). The effect of radiative cooling on the water uptake is more pronounced at higher $\mathrm{RH}$ since condensation starts to dominate the water capturing. A comparative field test conducted using two pieces of $30 \mathrm{wt} \% \mathrm{LiCl}$-cellulose composite, with one exposed to the sky and the other shielded by nontransmissive cardboard, also shows the radiative cooling effect on the enhancement of water uptake (Note S10, Fig. S17). 
In addition to the synergistic thermosorption effect that enhances water capture capacity at lower temperature, water capture kinetics is greatly expedited by dispersion, as indicated by the results of dynamic vapor sorption experiments (Fig. 4F and Note S11) and field tests that recorded water uptake vs. time curve (Fig. 4G). More specifically, in terms of the rate of water uptake, LiCl-cellulose composite has a synergistic higher rate of water uptake and outperforms the summation of $\mathrm{LiCl}$ powder and cellulose scaffold alone. We employed nitrogen sorption isotherms to determine the surface area of LiCl, cellulose scaffold, and LiCl-cellulose composite. The LiCl-cellulose composite has a Brunauer-Emmett-Teller (BET) equivalent surface area of $0.331-0.366 \mathrm{~m}^{2} \mathrm{~g}^{-1}$, while the values of cellulose scaffold and $\mathrm{LiCl}$ powders are 0.177 and $0.047 \mathrm{~m}^{2} \mathrm{~g}^{-1}$ respectively (Note S13). The impregnated $\mathrm{LiCl}$ is dispersed and attached to the large surface area of the cellulose scaffold, resulting in a much greater surface area for the composite enhances sorption kinetics. The derivative of the water uptake vs. time curve (Fig. S19) is computed to compare the rate of absorption of the three samples and supports this hypothesis (Fig. 4F). The LiCl-cellulose composite absorbed more water compared to the sum of the cellulose scaffold and the $\mathrm{LiCl}$ powder in the field test, which reinforces the initial hypothesis (Fig. 4G). To the best of our knowledge, the developed LiCl-cellulose composite shows higher water uptake capacity than most of the recently reported AWH sorbent materials under all weather conditions (8\%-90\% RH) (comparisons of which are shown in Fig. 4H-I).

Outdoor experiments are conducted under natural sunlight to characterize system performance in real-life scenarios. A batch-mode AWH device consisting of a thin-wall and clear acrylic container and a LiClcellulose composite with a dark substrate is prototyped (Fig. 5A, Note S14). During the nighttime, the lid of the acrylic container is opened, exposing the LiCl-cellulose composite (approximate dimension $10 \times 15$ $x 0.1 \mathrm{~cm}^{3}$, mass $1.5136 \mathrm{~g}$ ) to the sky and the composite captures water from the ambient air. During the daytime, the container is sealed. Driven by the solar heating, the water captured during the night evaporates and continuously condenses on the inner wall of the acrylic as the composite temperature increases. Then the liquid water flows down along the slope of the top container and accumulates in the corner.

An eight-day AWH was demonstrated on June 13th -14th and July 2nd -July 7th, 2021, where the discontinuation is due to weather disruption. Figure 5B exemplifies one day of water harvesting (June 13th ) where the ambient temperature, sample surface temperature, and dew point are monitored. The water uptake of $\mathrm{LiCl}$-cellulose composite consistently increases during the entire capturing process. The actual water uptake on the first day reaches $4.06 \mathrm{~kg} \mathrm{~kg}^{-1}, 13 \%$ lower compared to the ideal water uptake. Sunrise begins at $6: 16 \mathrm{AM}$, the outdoor temperature quickly increased from $22^{\circ} \mathrm{C}$ to $28^{\circ} \mathrm{C}$ and the $\mathrm{RH}$ decreased from 78-59\%. At 11:03 AM, the acrylic container is sealed and the water releasing process starts. Within $1 \mathrm{~min}$, the inner wall of the device starts to mist, and the transparent container becomes translucent after 5 minutes. The evaporated water gradually condensed on the inner wall of the device (Fig. S21B). Between 11:03 AM and 3:24 PM, the device harvests $3.65 \mathrm{~kg} \mathrm{~kg}^{-1}$ of liquid water, which is $86 \%$ of the captured water in the LiCl-cellulose composite. Figure $5 \mathrm{C}$ demonstrates the repeatability of the 
liquid water collection in the field test on the next seven consecutive days, where the highest water uptake is $5.97 \mathrm{~kg} \mathrm{~kg}^{-1}$ on July 6 th .

The ionic conductivity test shows that the quality of the collected water is better than tap water (Note S15). Capture-release cycles are repeated 20 times in lab tests. The water release is initiated by a solar simulator (Note S16). The composite is shown to be durable without notable degradation as revealed by the stable weight and the EDX mappings of chlorine in the LiCl-cellulose composite (Fig. 5E). All of the material characterizations and performance demonstrations confirm that the LiCl-cellulose composite is a potential candidate for achieving highly efficient AWH due to its distinct advantages of extremely high water sorption capacity at low humidity, relatively low desorption energy, fast water sorption-desorption kinetics, continuous sub-ambient cooling, and good cyclic stability. With a comprehensive understanding of the mechanisms and performance of LiCl-cellulose composite, especially its noticeable portability, scalability, and high water uptake, we then model and predict the potential daily water production on a global scale using the performance characterized in the lab test (Fig. 5D, Note S17). The modeling takes the global climate data from national centers for environmental prediction (NCEP) of the year 2020 (47) and interpolated the amount of daily water production solely based on the local relative humidity. Assuming a scaling factor of $1 / 3$ to exclude days of low daily clearness index and limited radiative cooling effect, there is still a non-zero annual production of clean water even in the most draught region. The maximum cumulative water production can reach $1200 \mathrm{~L} \mathrm{~m}^{-2}$ in some insular and peninsular areas where the humidity level is high, but the freshwater resource is scarce. The estimated monthly water production in typical arid cities from Jan. to Dec. is shown in Fig. 5F. As climate conditions differ city by city, the LiCl-cellulose composite can harvest four times more water from the atmosphere in Bamako than in Lanzhou, for example, during the humid summers. Figure 5G presents average monthly water production and corresponding averaged $\mathrm{RH}$ for the top eight most arid cities on the list. The average daily water production is $10.6 \mathrm{~L} \mathrm{~kg}^{-1}$ day $^{-1}$ in Phoenix, AZ, USA; therefore, a $3 \mathrm{~m}^{2}$ of the LiCl-cellulose composite is needed to meet the daily water usage need including cooking, drinking, and hygiene (48). The high performance LiCl-cellulose composite has the potential to mitigate the water shortage and the associated global challenges.

\section{Conclusion}

We demonstrate a full functionality and high-efficiency $\mathrm{AWH}$ by the passive radiative cooling LiClcellulose composite working across a wide range of $\mathrm{RH}\left(>8 \% \mathrm{RH}, 25^{\circ} \mathrm{C}\right)$ and elaborate the synergistic integration: 1) dispersed hygroscopic $\mathrm{LiCl}$ for moisture sorption and liquefaction, 2) multiscale cellulose structure for water storage and transport, 3) passive radiative cooling enhanced sorption and condensation with zero primary energy consumption, and 4) improved solar absorption by a dark substrate for rapid water release, i.e. temperature-swing strategy. The established theoretical understanding involving different sorption mechanisms, and radiative cooling effect is validated and well predicts the experimental water uptake and explains the synergy among the composite-water-energy interaction. Field tests demonstrate a water production rate of $>5.97 \mathrm{~L} \mathrm{~kg}^{-1}$ day $^{-1}$ under $0.9-1.1$ sun. 
These synergistic functions render an extraordinarily promising and scalable approach for an atmospheric water harvesting system with high production rate, cost-effectiveness, environmental friendliness, high stability, and completely safe water collection. The rational system design utilizing the multiscale structure of cellulose, synergistic effect of different mechanisms, and temperature-swing strategy paves the way for future advanced AWH systems.

\section{References}

1. M. M. Mekonnen, A. Y. Hoekstra, Four billion people facing severe water scarcity. Science advances 2 , e1500323 (2016).

2. M. Rezaei, A. Alsaati, D. M. Warsinger, F. Hell, W. M. Samhaber, Long-running comparison of feedwater scaling in membrane distillation. Membranes 10, 173 (2020).

3. D. M. Warsinger, S. Nejati, H. F. Juybari, Energy efficiency metrics in membrane distillation. Advances in Water Desalination Technologies. Singapore: World Scientific, 263-288 (2021).

4. H. B. Parmar et al., Nanofluids improve energy efficiency of membrane distillation. Nano Energy 88, 106235 (2021).

5. S. Cordoba, A. Das, J. Leon, J. M. Garcia, D. M. Warsinger, Double-acting batch reverse osmosis configuration for best-in-class efficiency and low downtime. Desalination 506, 114959 (2021).

6. B. Peñate, L. García-Rodríguez, Current trends and future prospects in the design of seawater reverse osmosis desalination technology. Desalination 284, 1-8 (2012).

7. L. Malaeb, G. M. Ayoub, Reverse osmosis technology for water treatment: state of the art review. Desalination 267, 1-8 (2011).

8. L. Shi, X. Wang, Y. Hu, Y. He, Y. Yan, Solar-thermal conversion and steam generation: a review. Applied Thermal Engineering 179, 115691 (2020).

9. Y. Shi, O. Ilic, H. A. Atwater, J. R. Greer, All-day fresh water harvesting by microstructured hydrogel membranes. Nature communications 12, 1-10 (2021).

10. H. Ghasemi et al., Solar steam generation by heat localization. Nature communications $5,1-7$ (2014).

11. X. Zhou, H. Lu, F. Zhao, G. Yu, Atmospheric water harvesting: a review of material and structural designs. ACS Materials Letters 2, 671-684 (2020).

12. A. Deepak, T. D. Wilkerson, L. H. Ruhnke, Atmospheric water vapor. (Elsevier, 2013).

13. D. Seo, J. Lee, C. Lee, Y. Nam, The effects of surface wettability on the fog and dew moisture harvesting performance on tubular surfaces. Scientific reports 6, 1-11 (2016).

14. O. Klemm et al., Fog as a fresh-water resource: overview and perspectives. Ambio 41, 221-234 (2012).

15. M. Zhou et al., Vapor condensation with daytime radiative cooling. Proceedings of the National Academy of Sciences 118, (2021). 
16. B. Khalil et al., A review: dew water collection from radiative passive collectors to recent developments of active collectors. Sustainable Water Resources Management 2, 71-86 (2016).

17. J. Xu et al., Efficient solar-driven water harvesting from arid air with metal-organic frameworks modified by hygroscopic salt. Angewandte Chemie International Edition 59, 5202-5210 (2020).

18. R. Li, Y. Shi, M. Wu, S. Hong, P. Wang, Improving atmospheric water production yield: Enabling multiple water harvesting cycles with nano sorbent. Nano Energy 67, 104255 (2020).

19. P. A. Kallenberger, M. Fröba, Water harvesting from air with a hygroscopic salt in a hydrogel-derived matrix. Communications Chemistry 1, 1-6 (2018).

20. F. Fathieh et al., Practical water production from desert air. Science advances 4, eaat3198 (2018).

21. H. Kim et al., Water harvesting from air with metal-organic frameworks powered by natural sunlight. Science 356, 430-434 (2017).

22. N. Hanikel et al., Evolution of water structures in metal-organic frameworks for improved atmospheric water harvesting. Science 374, 454-459 (2021).

23. F. Zhao et al., Super Moisture-Absorbent Gels for All-Weather Atmospheric Water Harvesting. Advanced Materials 31, 1806446 (2019).

24. C. Lei et al., Polyzwitterionic Hydrogels for Efficient Atmospheric Water Harvesting. Angewandte Chemie, (2022).

25. Y. Tu, R. Wang, Y. Zhang, J. Wang, Progress and expectation of atmospheric water harvesting. Joule 2, 1452-1475 (2018).

26. H. Lu et al., Materials Engineering for Atmospheric Water Harvesting: Progress and Perspectives. Advanced Materials, 2110079 (2022).

27. H. Shan et al., High-yield solar-driven atmospheric water harvesting with ultra-high salt content composites encapsulated in porous membrane. Cell Reports Physical Science 2, 100664 (2021).

28. M. Ejeian, A. Entezari, R. Wang, Solar powered atmospheric water harvesting with enhanced LiCl/MgSO4/ACF composite. Applied Thermal Engineering 176, 115396 (2020).

29. Price of LiCl. https://xingjiubio.en.made-in-china.com/product/idMtDcSohAUJ/China-Factory-BulkPrice-High-Purity-Lithium-Chloride-7447-41-8-with-Best-Price.html.

30. N. P. Service, Death Valley. https://www.nps.gov/deva/planyourvisit/weather.htm.

31. T. Li et al., Developing fibrillated cellulose as a sustainable technological material. Nature 590, 47-56 (2021).

32. V. E. Etuk, I. O. Oboh, B. R. Etuk, E. O. Johnson, K. Egemba, Nanocellulose: Types, Sythesis and Applications. In the European conference on sustainability, energy \& the environment 2018 official conference proceedings.

33. Y. Tian et al., Superhydrophobic and Recyclable Cellulose-Fiber-Based Composites for High-Efficiency Passive Radiative Cooling. ACS Applied Materials \& Interfaces 13, 22521-22530 (2021).

34. T. Li et al., A radiative cooling structural material. Science 364,760 (2019). 
35. G. Ulpiani, G. Ranzi, J. Feng, M. Santamouris, Expanding the applicability of daytime radiative cooling: Technological developments and limitations. Energy and Buildings 243, 110990 (2021).

36. M. R. Conde, Properties of aqueous solutions of lithium and calcium chlorides: formulations for use in air conditioning equipment design. International Journal of Thermal Sciences 43, 367-382 (2004).

37. E. Schindelholz, L.-k. Tsui, R. G. Kelly, Hygroscopic particle behavior studied by interdigitated array microelectrode impedance sensors. The Journal of Physical Chemistry A 118, 167-177 (2014).

38. S. Bouzenada, A. Kaabi, L. Fraikin, A. Léonard, in AIP Conference Proceedings. (AIP Publishing LLC, 2017), vol. 1809, pp. 020009.

39. Y. Zhang, R. Wang, T. Li, Y. Zhao, Thermochemical characterizations of novel vermiculite-LiCl composite sorbents for low-temperature heat storage. Energies 9, 854 (2016).

40. M. Wang et al., Solar-powered nanostructured biopolymer hygroscopic aerogels for atmospheric water harvesting. Nano Energy 80, 105569 (2021).

41. F. Gong et al., Agricultural waste-derived moisture-absorber for all-weather atmospheric water collection and electricity generation. Nano Energy 74, 104922 (2020).

42. T.-D. Nguyen, E. Sierra, H. Eguiraun, E. Lizundia, Iridescent cellulose nanocrystal films: the link between structural colour and Bragg's law. European Journal of Physics 39, 045803 (2018).

43. A. P. Raman, M. Abou Anoma, L. Zhu, E. Rephaeli, S. Fan, Passive radiative cooling below ambient air temperature under direct sunlight. Nature 515, 540-544 (2014).

44. P. Robinson, J. Davies, Laboratory determinations of water surface emissivity. Journal of Applied Meteorology 11, 1391-1393 (1972).

45. Z. Pan et al., The upside-down water collection system of Syntrichia caninervis. Nature plants 2, 1-5 (2016).

46. M. Dubois et al., Investigation of the H2O-NaCl-LiCl System: A Synthetic Fluid Inclusion Study and Thermodynamic Modeling from $-50^{\circ}$ to $+100^{\circ} \mathrm{C}$ and up to $12 \mathrm{~mol} / \mathrm{kg}$. Economic Geology 105 , 329-338 (2010).

47. S. Saha et al. (Research Data Archive at the National Center for Atmospheric Research, Computational and Information Systems Laboratory, Boulder, CO, 2011).

48. B. R. a. B. Reed, "How much water is needed in emergencies," (World Health Organization, 2004).

\section{Figures}




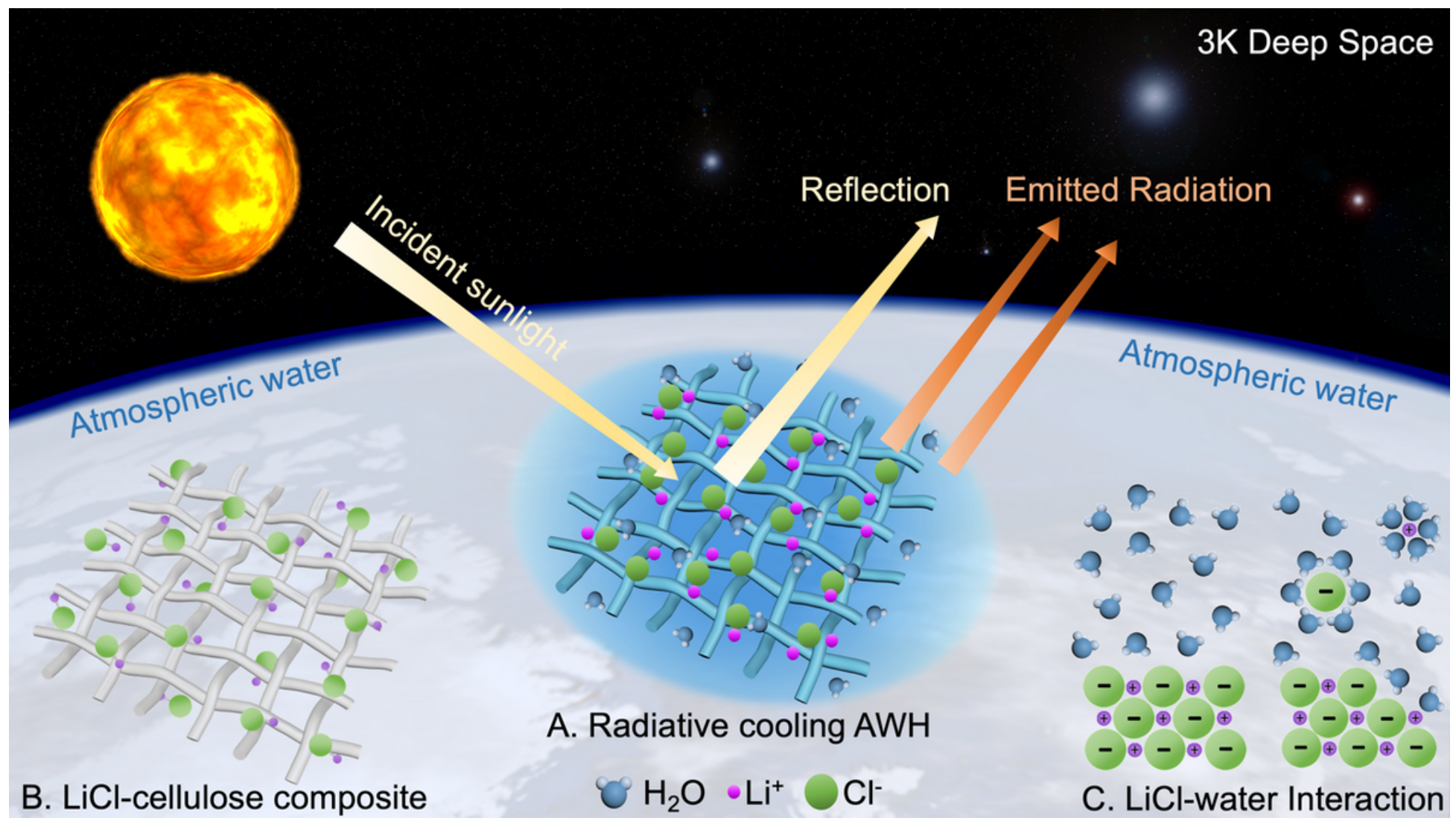

Figure 1

LiCl-cellulose composite demonstrates energy-efficient atmospheric water harvesting and collection. (A) Schematic of mechanisms of atmospheric water harvesting by radiative cooling LiCl-cellulose composite. (B) Cooler scaffolds of LiCl-cellulose composite and complex networks show strong physical entanglements and water storage capabilities. (C) Molecular interaction between $\mathrm{LiCl}$ and water. 
A
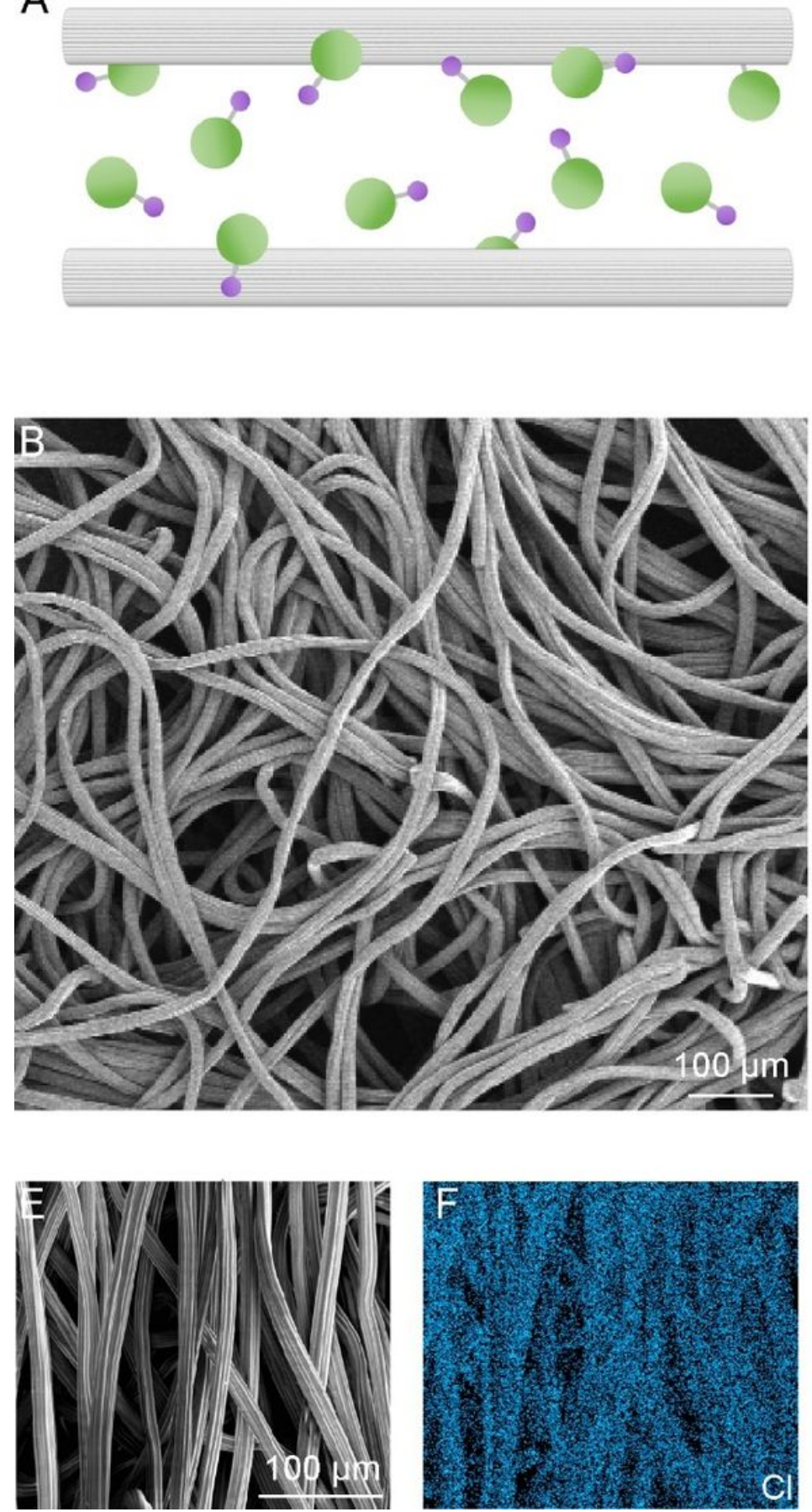

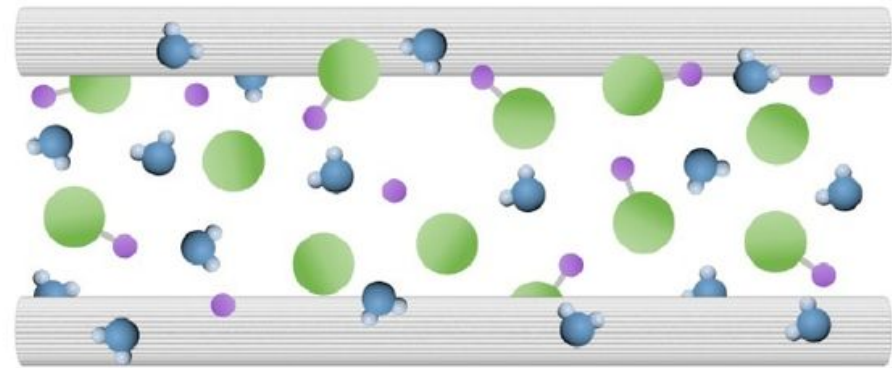

$\mathcal{O} \mathrm{H}_{2} \mathrm{O} \bigcirc \mathrm{LiCL} \mathrm{Cl}^{-} \odot \mathrm{Li}^{+}$
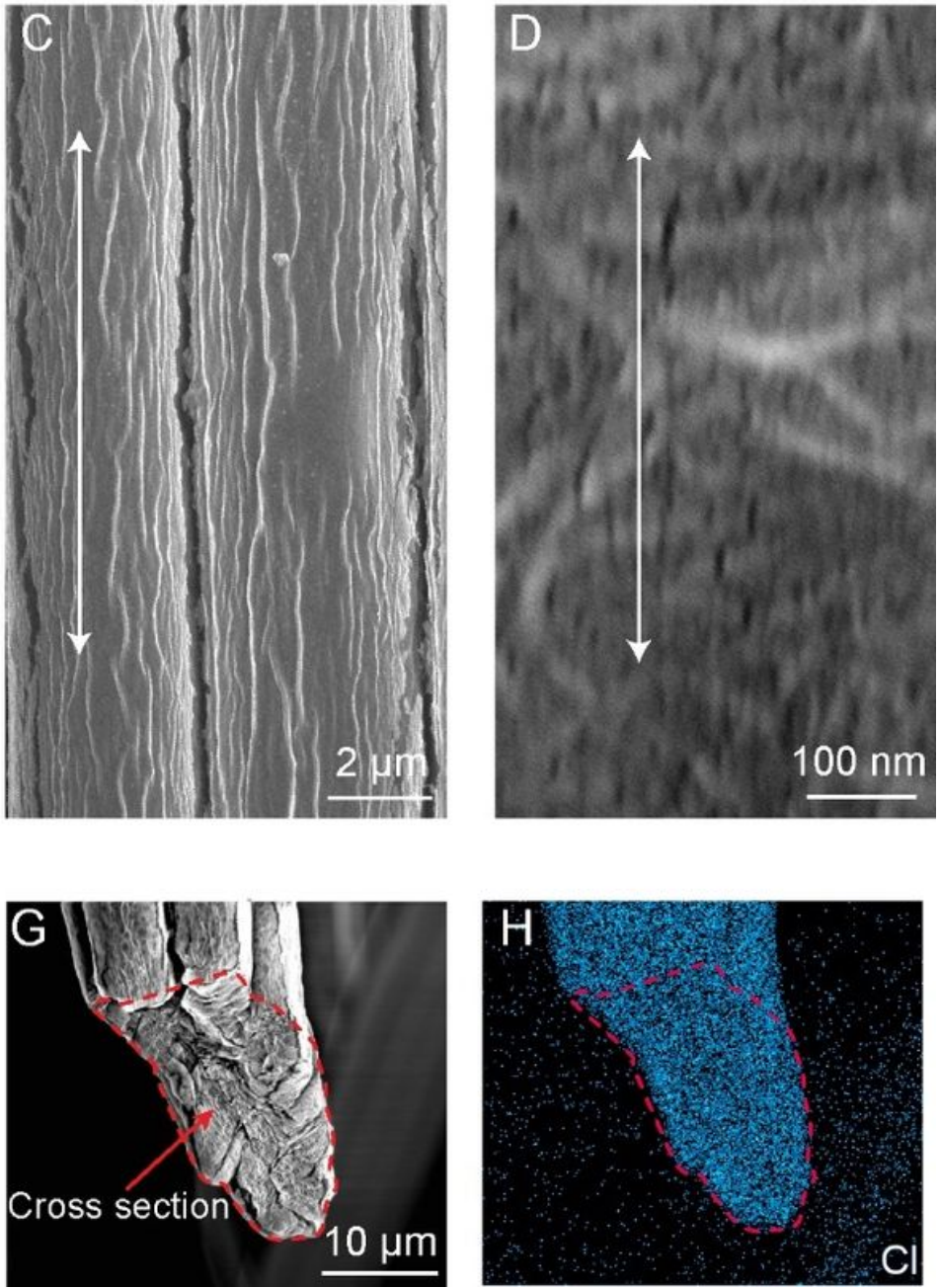

Figure 2

Synthesis and morphology of the LiCl-cellulose composite. (A) The schematics of the LiCl ion intercalation process. (B) SEMs of LiCl-cellulose composite composed of entangled cellulose fiber bundles. (C) A magnified view of the cellulose fiber surface. (D) Partially aligned cellulose nanofibers. (E) Cellulose fiber network. (F) The corresponding EDX mapping of $\mathrm{Cl}$ shows the coating uniformity. (G) Cross-section view of the cellulose fiber bundle. $(\mathrm{H})$ The corresponding $\mathrm{Cl}$ mapping demonstrates that $\mathrm{LiCl}$ is impregnated into the fibers by intercalation. 
E
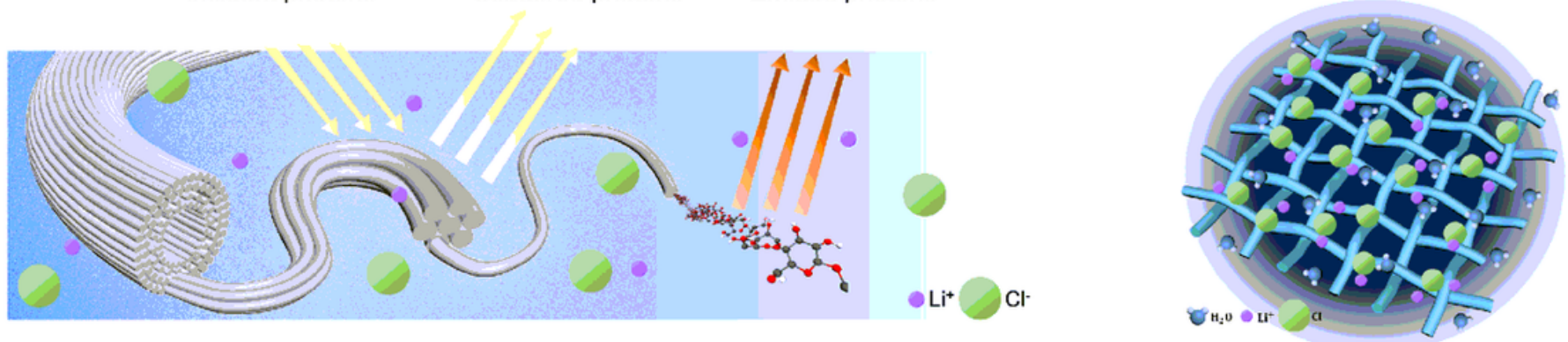

Fiber bundle

Microfibers

Nanofibers

Molecular chain

B

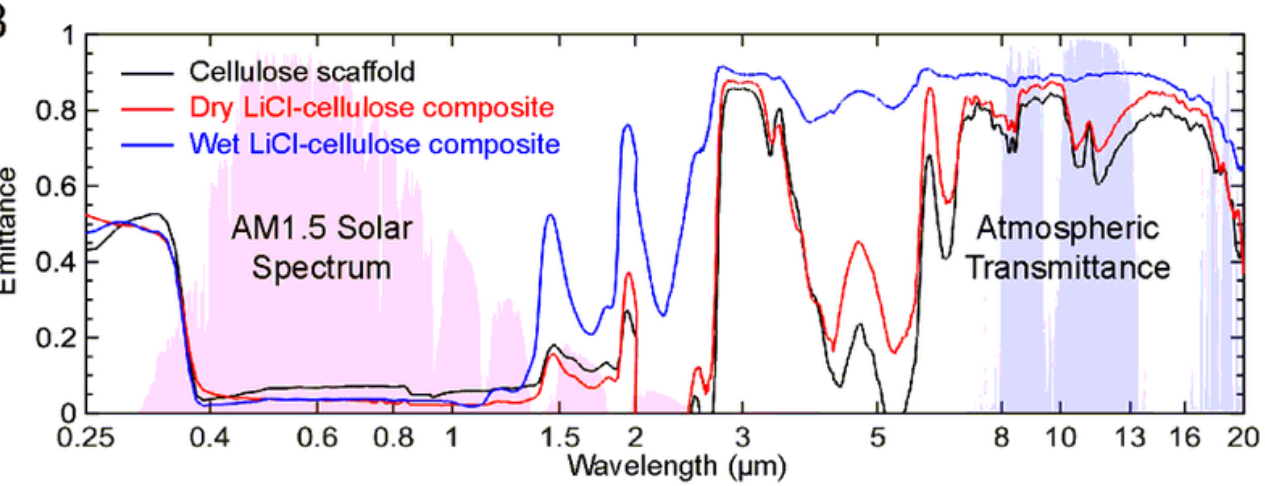

F

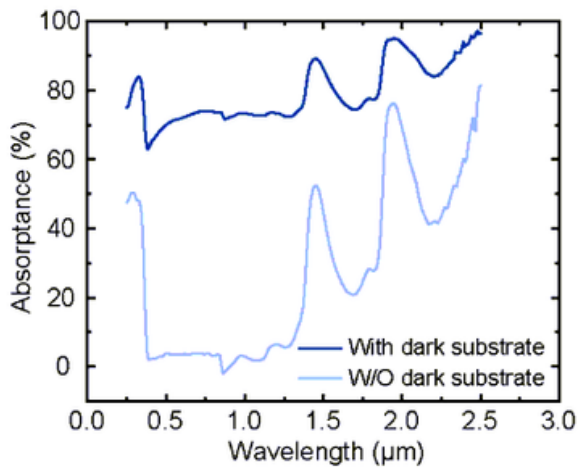

C

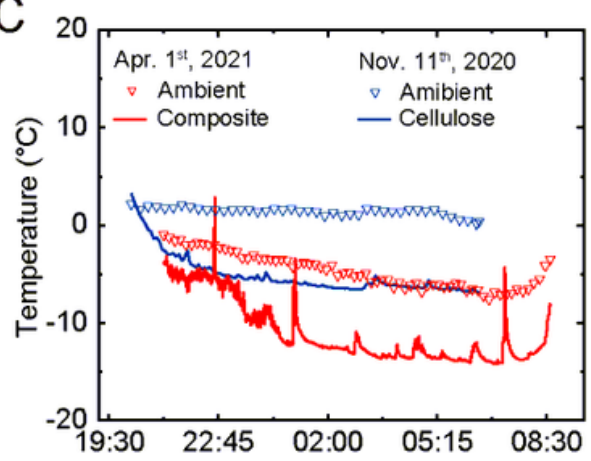

D

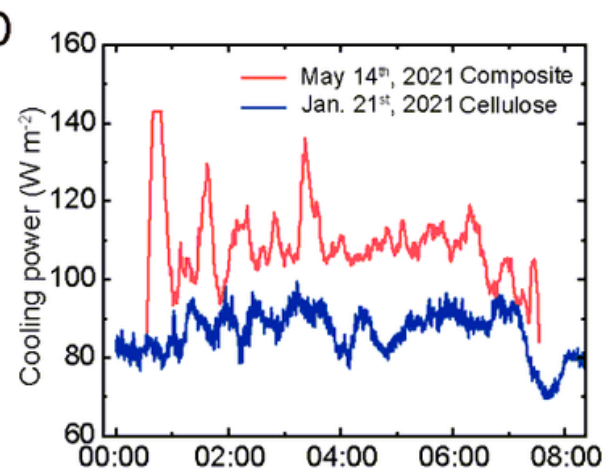

G

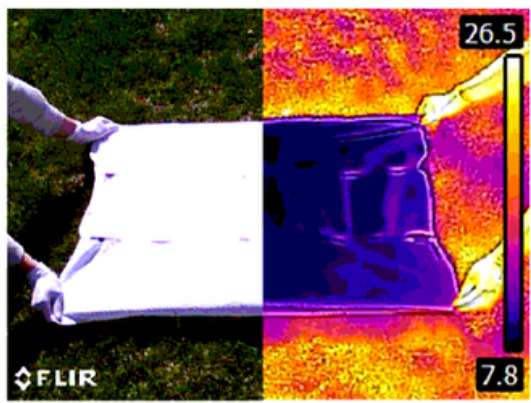

Figure 3

Optical and thermal characterization of LiCl-cellulose composite. (A) Schematic of radiative cooling effects on the LiCl-cellulose composite. (B) Emissivity in the ultraviolet, visible, and infrared range of the cellulose and LiCl-cellulose composite in dry and wet conditions. (C) Profiles of the sample surface temperatures and ambient temperature of cellulose scaffold and LiCl-cellulose composite during two different nights. (D) Profiles of the cooling power measured for the cellulose scaffold and LiCl-cellulose composite during two different nights. (E) Schematic of water extraction from the LiCl-cellulose composite with an underneath dark substrate. (F) Absorptance spectra of LiCl-cellulose composite with and without dark substrate. (G) Macroscopic and IR photo of a large-scale LiCl-cellulose composite on Apr. $30^{\text {th }}, 2021$. The ambient temperature is $21^{\circ} \mathrm{C}$. 
A

Hydrogen bonding/solvation
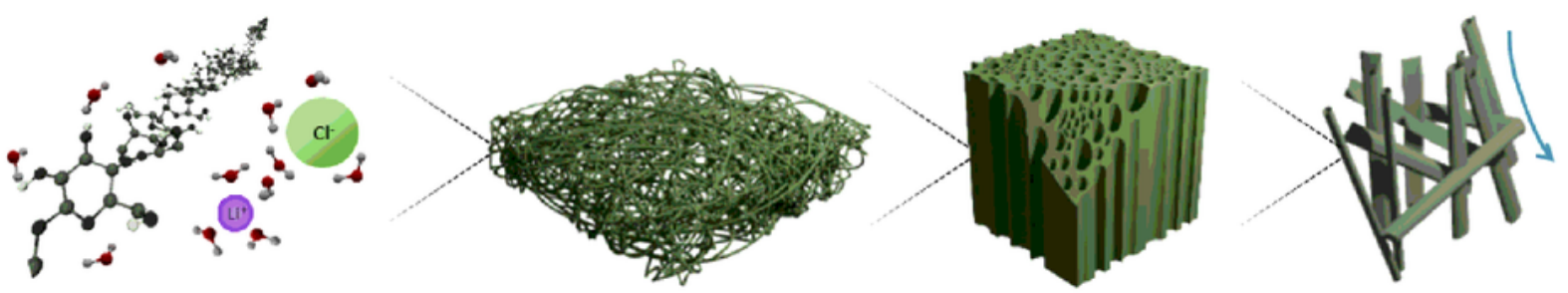

Molecular

(A)

B

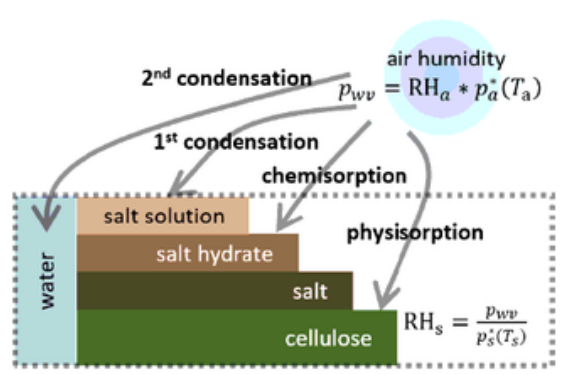

E

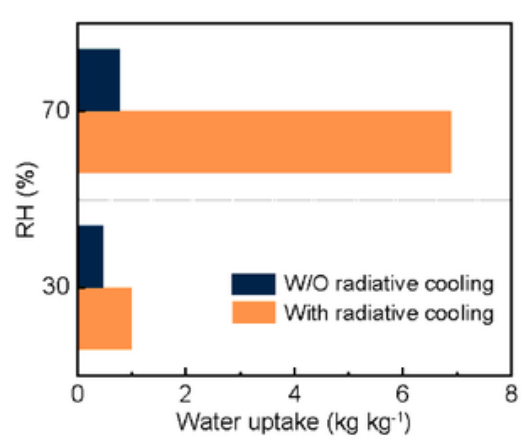

Nanoporous

$(\mathrm{nm})$

C

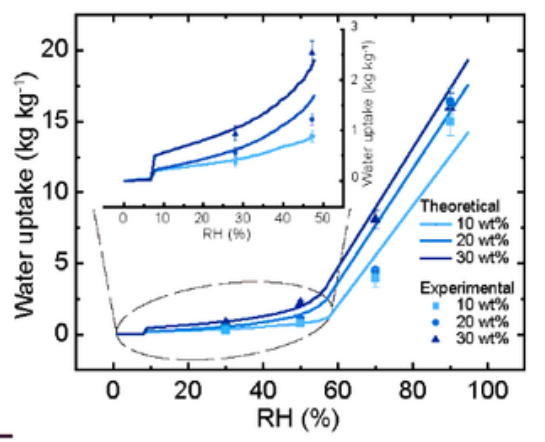

F

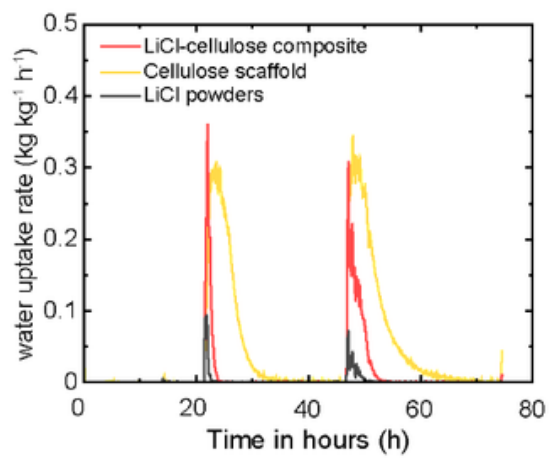

Meso-/Macroporous $(\mathrm{nm}-\mu \mathrm{m})$
Fiber network $(\mathrm{mm})$

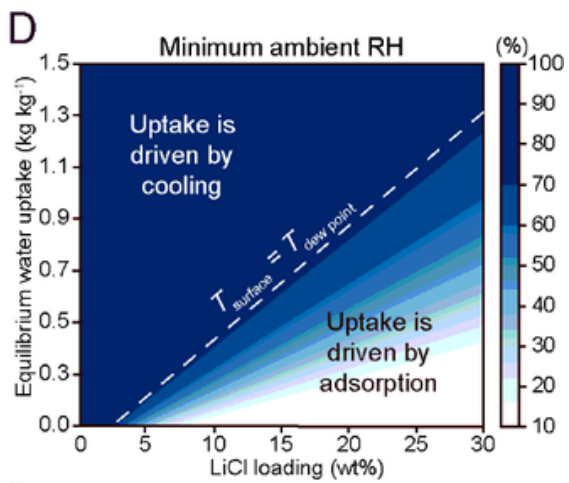

G
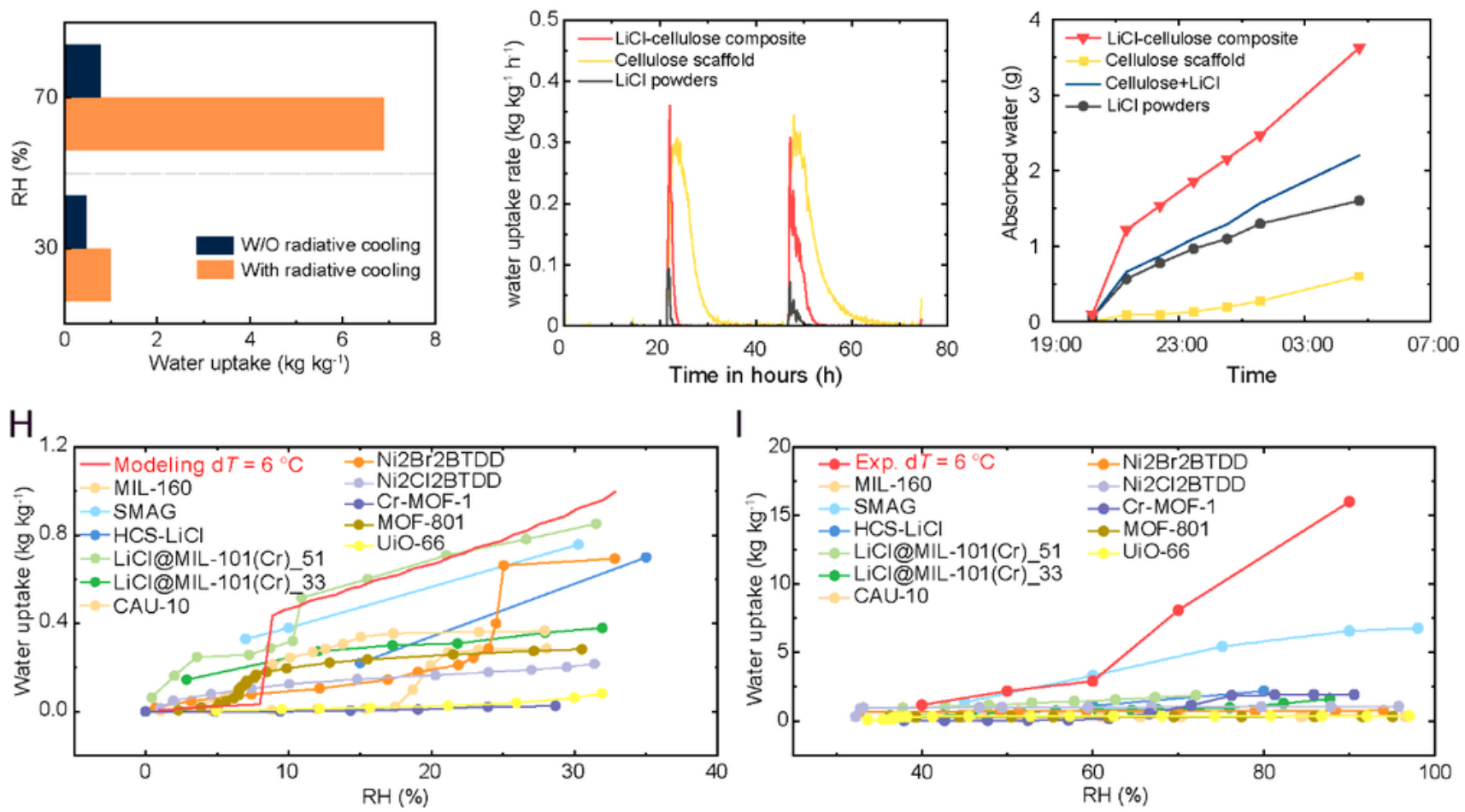

Figure 4

Synergistic effects among radiative cooling, hygroscopic LiCl, and cellulose scaffold. (A) Schematic of LiCl-cellulose composite system. (B) Experimental and theoretical water uptake of LiCl-cellulose composite samples (10-30 wt\%) with radiative cooling. (C) Experimental and theoretical water uptake of LiCl-cellulose composite samples with 10-30 wt\% at 0-100\% RH. Inset is an enlargement of the experimental and theoretical water uptake from $0-50 \% \mathrm{RH}$. (D) Maximum water uptake as function of RH 
for $6{ }^{\circ} \mathrm{C}$ below the $25^{\circ} \mathrm{C}$ ambient temperature. (E) Comparison of water uptake of LiCl-cellulose composite with and without radiative cooling effect at $30 \%$ and $70 \% \mathrm{RH}$. The LiCl-cellulose composite has a $30 \mathrm{wt} \%$ loading. (F) The rate of change of water uptake of cellulose, $\mathrm{LiCl}$, and $\mathrm{LiCl}$-cellulose composite samples based on sorption isotherm characterization. $(\mathrm{G})$ Collected water mass as a function of time during the field test of cellulose scaffold, $\mathrm{LiCl}$ powder, and LiCl-cellulose composite. $(\mathrm{H})$ Water uptake comparison with other sorption materials for $\mathrm{AWH}$ at the lower end of the RH spectrum. $\mathrm{d} T$ is sample temperature below the ambient temperature. (I) Water uptake comparison with other sorption materials for $\mathrm{AWH}$ at the higher end of the $\mathrm{RH}$ spectrum.

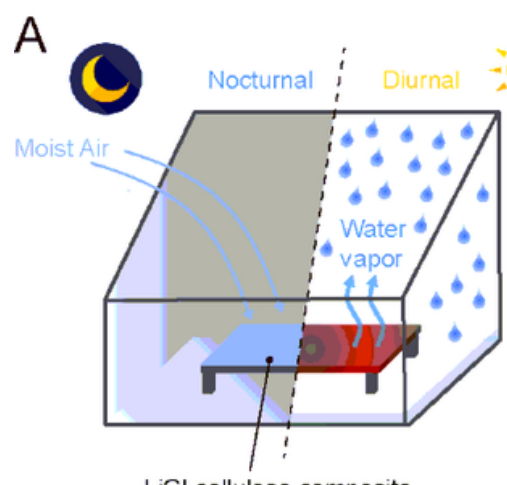

$\mathrm{D}$

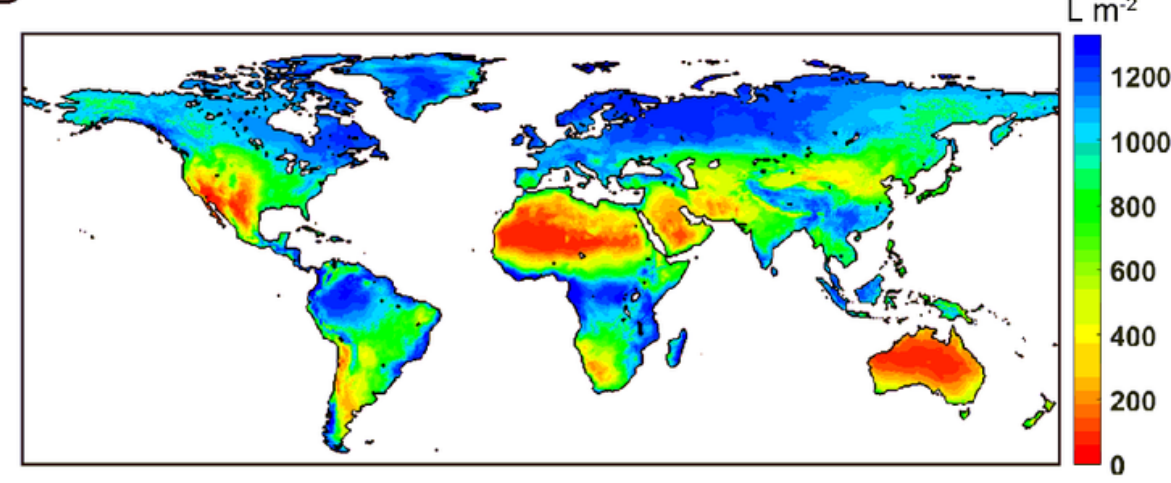

$\mathrm{F}$

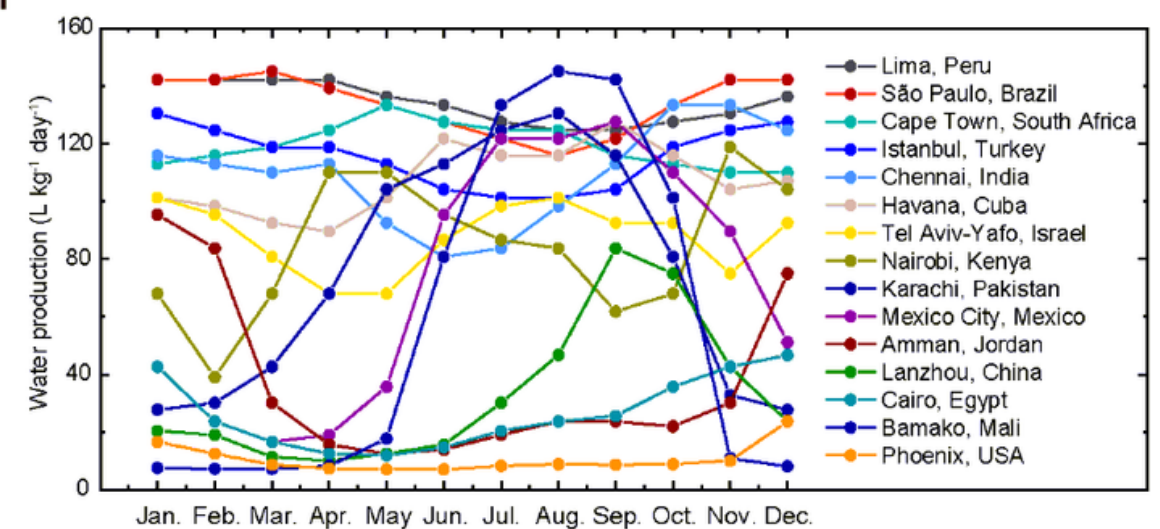

C

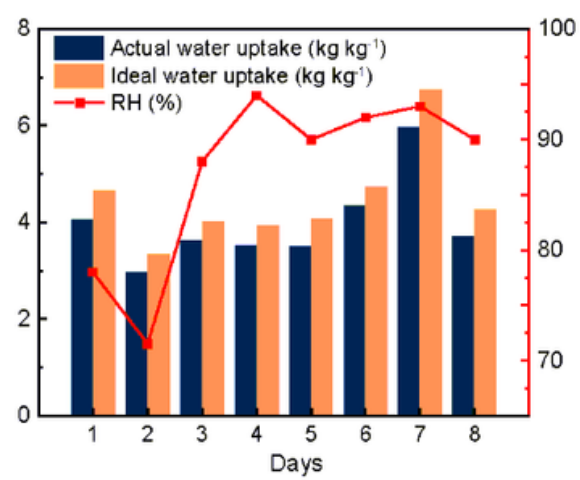

E

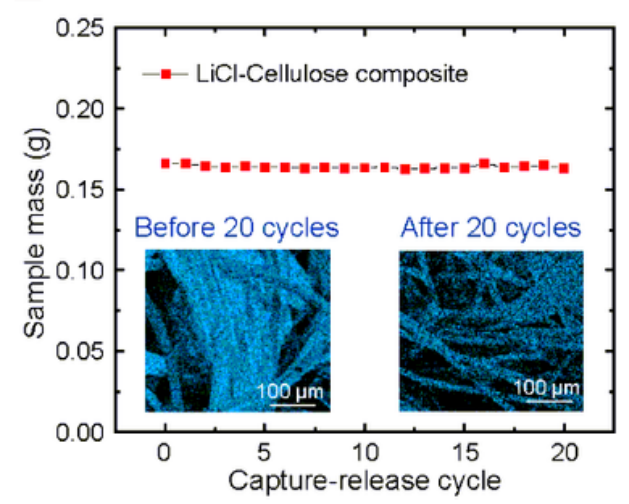

G

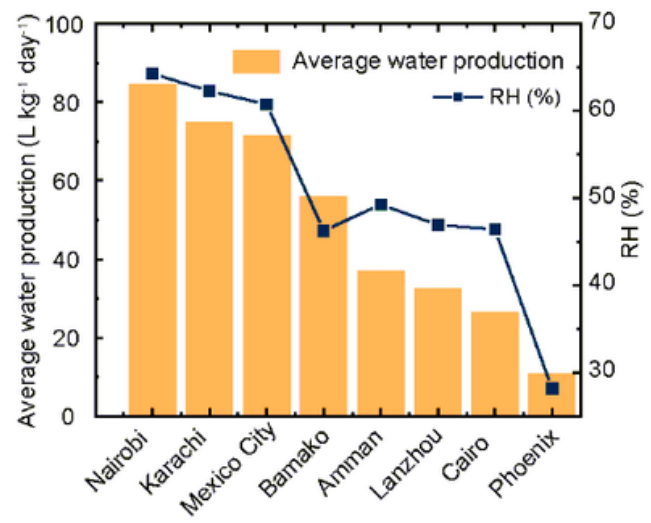

\section{Figure 5}

Scalability, impact, and the prospect of AWH by LiCl-cellulose composite. (A) Schematic of the AWH device with the LiCl-cellulose composite. (B) Temperature and water uptake of a $30 \mathrm{wt} \% \mathrm{LiCl}$-cellulose composite during a field test. (C) Field test results of 8 days including ideal and actual water uptake in $\mathrm{kg}$ 
$\mathrm{kg}^{-1}$. The actual water uptake is defined as the mass of water released over the initial mass of composite. The ideal water uptake is defined as the mass of water captured over the initial mass of the composite. (D) The predicted annual water collection per area based on local climate around the globe. (E) Dry mass of $30 \mathrm{wt} \% \mathrm{LiCl}$-cellulose composite during 20 capturing-releasing cycles, which exhibit the $\mathrm{LiCl}$ is stable and will be retained in the composite for a durable application. The inset EDX mappings are of chlorine in the composites before and after 20 cycles. (F) Estimated average monthly water production in arid cities around the world. (G) Relative humidity and average water production for the eight most arid cities from (F).

\section{Supplementary Files}

This is a list of supplementary files associated with this preprint. Click to download.

- SI.docx 